\title{
Pulse Edge-Only Signaling Method Comparison for Wireline Interconnects
}

\author{
Dmitriy Garmatyuk, ${ }^{1}$ Kyle Kauffman, ${ }^{2}$ and Andrew Martwick ${ }^{3}$ \\ ${ }^{1}$ Department of ECE, Miami University, Oxford, OH, USA \\ ${ }^{2}$ Air Force Institute of Technology, WPAFB, OH 45433, USA \\ ${ }^{3}$ Intel Corporation, Portland, OR, USA \\ Correspondence should be addressed to Dmitriy Garmatyuk; garmatd@miamioh.edu
}

Received 30 April 2014; Revised 25 July 2014; Accepted 4 August 2014; Published 14 September 2014

Academic Editor: Changhwan Shin

Copyright (C) 2014 Dmitriy Garmatyuk et al. This is an open access article distributed under the Creative Commons Attribution License, which permits unrestricted use, distribution, and reproduction in any medium, provided the original work is properly cited.

\begin{abstract}
Typical high-speed electrical transmission lines use a variety of precoding and equalization techniques to counter the frequencydependent channel loss and environmental conditions such as ISI. In this paper, we suggest a relatively narrow-band signaling method that is resilient to the effects of ISI and crosstalk and can be implemented with existing technology. Alternative modulation schemes are analyzed in terms of effectiveness, performance, and cost. In particular, line-encoded and on-off keyed modulation methods are evaluated in simulations of transmission lines to gauge effectiveness in high-speed conditions with limiting ISI.
\end{abstract}

\section{Introduction}

Modern digital communications across transmission lines have long been analyzed for methods to generate and transmit binary signals at high speeds. The most popular technique is to transmit using the non-return-to-zero (NRZ) line code modulation scheme and subsequently use a variety of channel equalization methods to counteract channel distortions such as intersymbol interference (ISI) $[1,2]$. Such equalization methods, most recently, were focused on adaptive filter design to cancel out the channel effects estimated via, for example, time-domain reflectometry (by evaluating channel's impulse response). In [3] the authors suggest generating error terms derived from the channel's impulse response to be used in equalizing filter which minimizes ISI accumulation. The method showed promise in simulations up to $20 \mathrm{~Gb} / \mathrm{s}$ over 6in wireline channels. Another filter-based method described in [4] specifically addresses the problem of delay line usage in such systems, which are prone to process, voltage, and temperature variations. By proposing an active delay line the authors claim improvement over these shortcomings and demonstrate the experimental performance at $10 \mathrm{~Gb} / \mathrm{s}$ over 20 in interconnect. The system required four buffers used as delay line components and four buffers used as amplifiers.
Simulation study of another FIR-based equalizer in [5] used 7-tap delay line model with passive LC components, which would require significant chip area for implementation. FIR equalizers implemented in GaAs HBT and 0.18 um CMOS to achieve $5 \mathrm{~Gb} / \mathrm{s}$ over 26 in FR4 backplane with active NEXT cancellation (also via FIR implementation) are described in [6]. The overview of passive and active equalization methods is also given in [7]. These are fairly complex equalization methods which tend to increase the device power consumption, reduce compatibility with other devices (via, e.g., increasing crosstalk if high-frequency components of squarepulse signals are boosted), and add cost.

In this work we propose a different approach to equalization: via introducing a square-pulse-compatible signaling technique which utilizes square-pulse edges only. With bit widths at or below 100 ps the attractiveness of square pulses and corresponding "eye-diagram" based method of their recovery in the receiver diminishes, as signal distortions brought about by ISI, as well as channel attenuation, resonances of the interconnect, and external noise significantly degrade receiver's ability to correctly detect data. Figure 1 shows a simulated NRZ-encoded signal generated at $5 \mathrm{~Gb} / \mathrm{s}$ and $20 \mathrm{~Gb} / \mathrm{s}$ and passed through a low-pass channel with a $3 \mathrm{~dB}$ cutoff around $8 \mathrm{GHz}$. The graphs show that the signal 


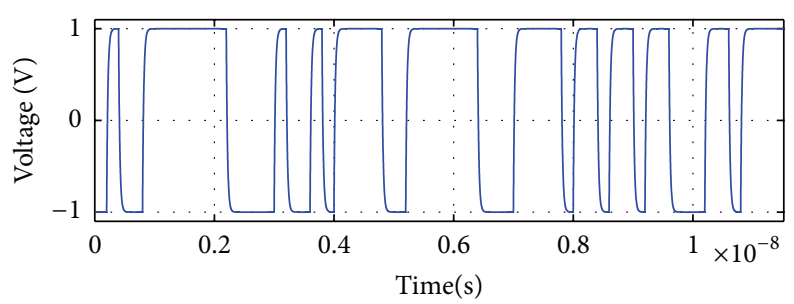

(a)

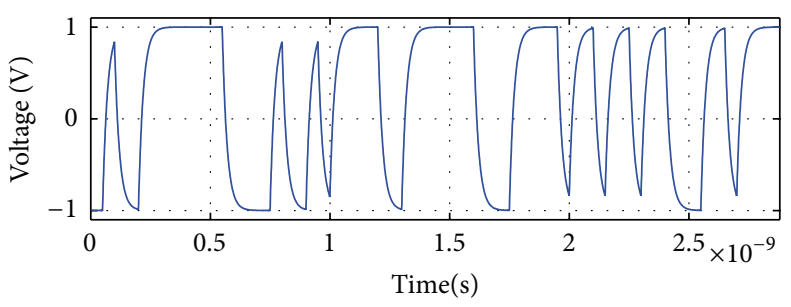

(b)

Figure 1: $5 \mathrm{~Gb} / \mathrm{s}$ (a) and $20 \mathrm{~Gb} / \mathrm{s}$ (b) signals sent through LPF channel.

distortion due to channel's low-pass characteristics becomes more severe as the required data rate increases. In order to allow for accurate data transmission at higher bit rates, new approaches must be explored that can deal with the worsening channel loss at higher frequencies where ordinary approaches fail. Other channel distortions of significance include jitter and crosstalk, which limit signal data rate $[8,9]$; in this paper we will focus on the effects of external noise (AWGN) and ISI.

The paper is organized as follows. Section 2 introduces two types of square-pulse receiver models used in subsequent benchmark simulations, matched filter and eye-diagram based model, and also describes another benchmark signaling method, on-off keying (OOK), along with stating comparison techniques between them and the proposed method. Section 3 introduces edge-only transmission method and illustrates its transmitter and receiver concepts. Section 4 shows and discusses simulation results for all types of considered signaling methods and receiver models. Conclusions are presented then in Section 5.

\section{Wireline Communication System Performance}

This section analyzes various methods of transmission. For all methods, the input data will be in the same format; we will receive a square pulse waveform which represents each symbol intended to be transmitted. For simulation purposes the input will be discretized into an $n$ bit vector I, each representing the value of a single bit, where the input buffer of bits to be sent is $n$. That is,

$$
\mathbf{I}[k]=\{0,1\}, \quad 0<k<n .
$$

This vector will be modulated with one of the following methods.
2.1. Square Pulse Transmission. The most common form of signaling on wireline transmissions is via NRZ square wave signals time-varying between a high and low voltage [1]. The simulated transmitter creates a set of $n_{1} \geq n$ samples at a sampling frequency $F$. This is done by interpolating the vector I onto the samples at even intervals. Thus, we have

$$
\mathrm{SQ}[k]=\mathbf{I}\left(\left\lfloor k \frac{n}{n_{1}}\right\rfloor\right), \quad 0 \leq k \leq n_{1} .
$$

If SQ[0] is sampled at time $t=0$, then, for a chosen $F$, sample $k$ occurs at time $k / F$. There are two receiver types that will be considered for this type of modulation, an ideal matched filter and an eye-diagram based receiver. The matched filter will produce response $O_{\text {match }}^{\prime}$ by correlating reference pulse with the input square wave I. Let $r$ be the number of samples per bit and let $Y[n]$ be the recovered samples at the receiver. Then matched filter result for some bit $b$ is

$$
O_{\text {match }}^{\prime}[n]=\int_{r b}^{r(b+1)} Y[n] d n,
$$

which in the discrete form is calculated using weighted summation. If we let the weight for our simulated samples equal 1 , then we simply have

$$
O_{\text {match }}^{\prime}[n]=\sum_{n=r b}^{r(b+1)} Y[n] .
$$

The resulting signal must be larger than a threshold value $T$ which depends on the receiver implementation. Thus, if $O_{\text {match }}^{\prime} \leq T$, the recovered bit $O_{\text {match }}$ is a 0 ; otherwise, it is 1.

The eye-diagram based receiver will instead sample the bit sometime within the interval $r b$ and $r(b+1)$. For simulation purposes, the window of sampling is $1 / 6$ of the total bit width and the sampled value must be above a threshold $T$ which 
is set to .1; then the recovered symbol $n$ for the eye-diagram based receiver is

$$
\begin{aligned}
O_{\text {eye }}^{\prime}[n]=Y[n] \mid n \in & {\left[\frac{r b+r(b+1)}{2}, \frac{r b+r(b+1)}{2}\right.} \\
& \left.+\frac{1}{3}(r b+r(b+1))\right] .
\end{aligned}
$$

That is, the recovered value $O_{\text {eye }}^{\prime}$ can be the value of any sample within $1 / 3$ of a symbol width from the midpoint of that symbol. If $O_{\text {eye }}^{\prime} \geq T$, then a 1 is recovered and a 0 is recovered otherwise.

2.2. On-Off Keying (OOK). This digital modulation scheme in its simplest form transmits a 0 symbol as a grounded line $(V=0)$ and a 1 symbol as a sinusoid at some carrier frequency $\omega$. It is equivalent to AM modulation where the only values for the coefficient of the sinusoid are 0 and 1 , which correspond to the value of a particular symbol. For simulation, the transmitter must generate from the input vector $\mathbf{I}$ a set of $n_{2}$ samples with a sampling frequency $F$ by amplitude modulating a sinusoid with the input bits I. However, since $n \neq n_{2}$ we need to stretch the input vector onto the number of samples generated. Formally, we have

$$
\operatorname{OOK}[k]=\mathbf{I}\left[\left\lfloor k \frac{n}{n_{2}}\right\rfloor\right] \sin \omega t, \quad 0 \leq k \leq n_{2} .
$$

If OOK[0] is sampled at time $t=0$, then for a chosen $F$ sample $k$ occurs at time $k / F$. The receiver consists of a simple energy detector. In simulation this can be taken as the sum of the absolute values of the bits within a given bit period and a check to see if this sum is above a threshold value T. Formally, let $r$ be the number of samples per bit and let $y$ be the signal incident at the receiver. Then matched filter result for some bit $b$ is

$$
O_{\text {on off }}^{\prime}=\sum_{i=r b}^{r(b+1)}|y[i]|^{2}
$$

and the recovered bit $O_{\text {on off }}=1$ if $O_{\text {on off }}^{\prime} \geq 1$; otherwise, $\mathrm{O}_{\text {on off }}=0$.

\subsection{Low-Pass Filtering Effect on Square Pulse and OOK} Signals. For square-pulse signaling, as data is transmitted at faster rates, the pulses will become narrower, as the period of each bit is $1 /$ (data rate). We know that signals at higher data rates have narrower square pulses and thus wider bandwidth; this is a result of the well-known Fourier transform (FT) pair:

$$
\operatorname{rect}\left(\frac{t}{\tau}\right) \underset{\tau, f}{\Longleftrightarrow}|\tau| \operatorname{sinc}(\tau f)
$$

As data rate increases, a transmitted bit will become narrower in the time domain and the effects of ISI will also become more pronounced.
In $\mathrm{OOK}$, the center frequency component will depend on the carrier frequency used to generate the sinusoid during the "high" bits. Most of the OOK signal's energy is concentrated around the carrier frequency; however, the rectangular windowing of the sinusoid with a $\operatorname{rect}(t)$ ensures that the energy will be spread out, just as with the square wave. Figure 2 shows passing a single bit through the same lowpass filter (LPF) using two different data rates. In simulation a $25 \mathrm{GHz}$ carrier was used and the bit rates are $2 \mathrm{~Gb} / \mathrm{s}$ and $10 \mathrm{~Gb} / \mathrm{s}$.

Notice that the signal after LPF tends to be above 0 when transmitting a "high" and at or around $V=0$ when transmitting a 0 . We can use this heuristic in construction of our on-off keying receiver by detecting a 1 if the energy detector's output is above a certain threshold value $T>0$, and 0 otherwise.

2.4. Comparison Techniques and Benchmarks. In order to compare the performance of signaling methods we will create tests for the performance in high noise environments and another for performance in high ISI environments. The noise environment will be simulated by injecting additive white Gaussian noise (AWGN) into the input signal before it is sent through the channel. Let $R[k]$ be an $n$-bit vector of unit-variance Gaussian random numbers, where $n$ is equal to the number of samples in the input signal $I[k]$. Then, for a constant $C$ which sets the noise intensity, we have

$$
I^{\prime}[k]=C R[k]+I[k] .
$$

The resultant vector $I^{\prime}$ will then be simulated to pass through the channel and will go through simulated recovery. The signal-to-noise ratio (SNR) of $I^{\prime}$ is defined to be

$$
\mathrm{SNR}=\frac{\operatorname{Energy}(I)}{\operatorname{Energy}(C R)},
$$

where the energy of a vector $\mathbf{V}$ is defined as the unnormalized quantity $\operatorname{Energy}(\mathbf{V})=\sum_{k \in \mathbf{V}} k^{2}$. The performance measure will be the bit error rate (BER), which is defined as number of bits errors/number of bits transmitted. Each modulation scheme will have $10^{4}$ iterations performed for a particular value of $C$, and $C$ was simulated for values between 0 and 2 (step size of .001). The Python pseudocode example as seen in Pseudocode 1 demonstrates the procedure used. The number of transmitted bits is calculated as the length of each bit vector (128 bits) minus the 7 bit lead-in and lead-out used for zero padding. The simulate function (Section 4 ) sets $C$ to the value of its only parameter and outputs the signal samples, output bit patterns, number of errors, SNR, and energy of the noise in that order, as seen below. Signals 0,1 , and 2 represent NRZ square pulse signaling, edge-only transmission (described in detail in Section 3), and OOK, respectively.

In addition, we will compare each signaling method against the others in the presence of ISI due to low-pass characteristics of the channel. We define a new function simulate $_{l p f}$ (Section 4) which takes as its input the value of the RC constant of the modeled channel and returns 

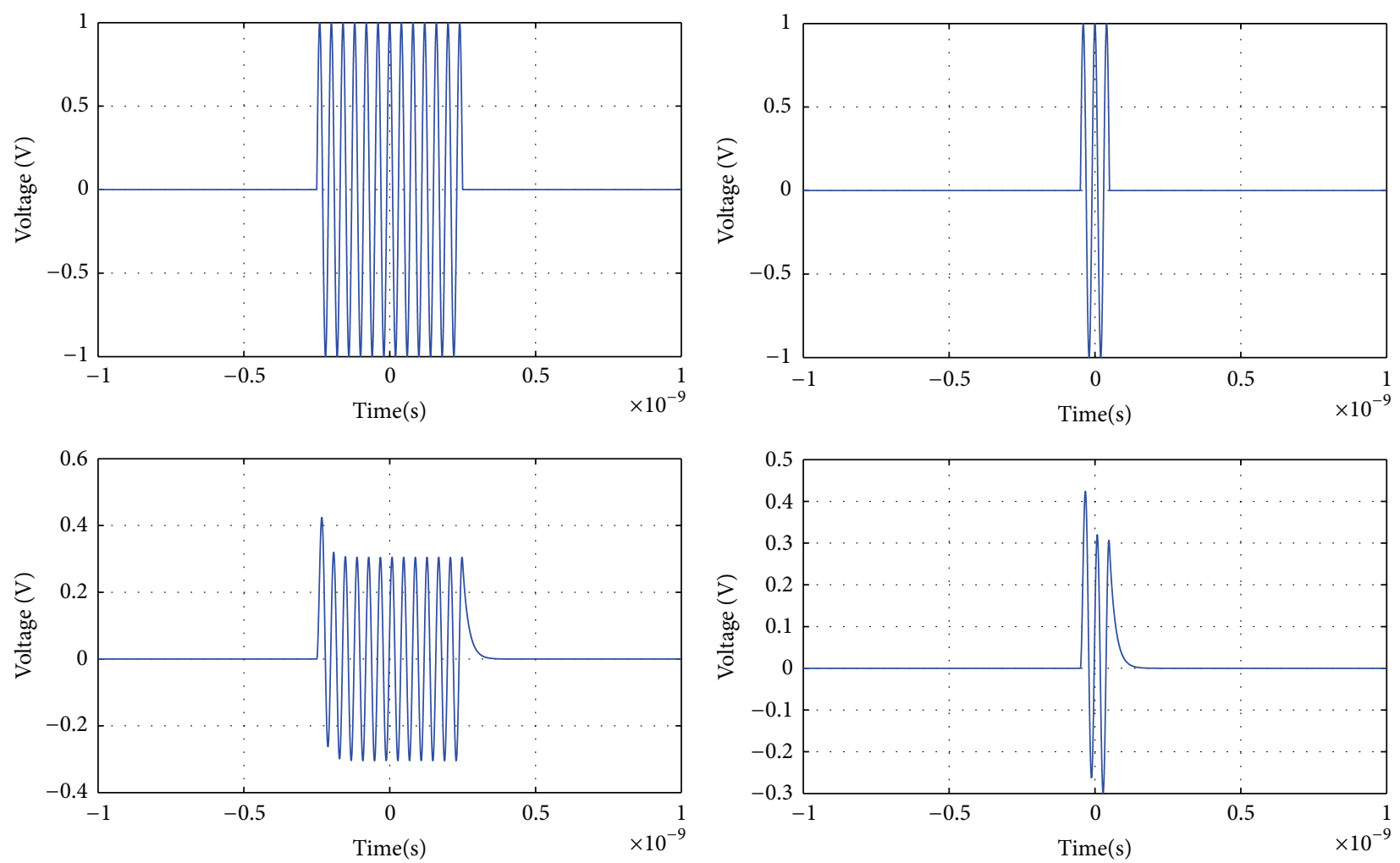

(a)

(b)

Figure 2: (a) $2 \mathrm{~Gb} / \mathrm{s}$ signal and output from LPF below and (b) $10 \mathrm{~Gb} / \mathrm{s}$ signal and its output from LPF below.

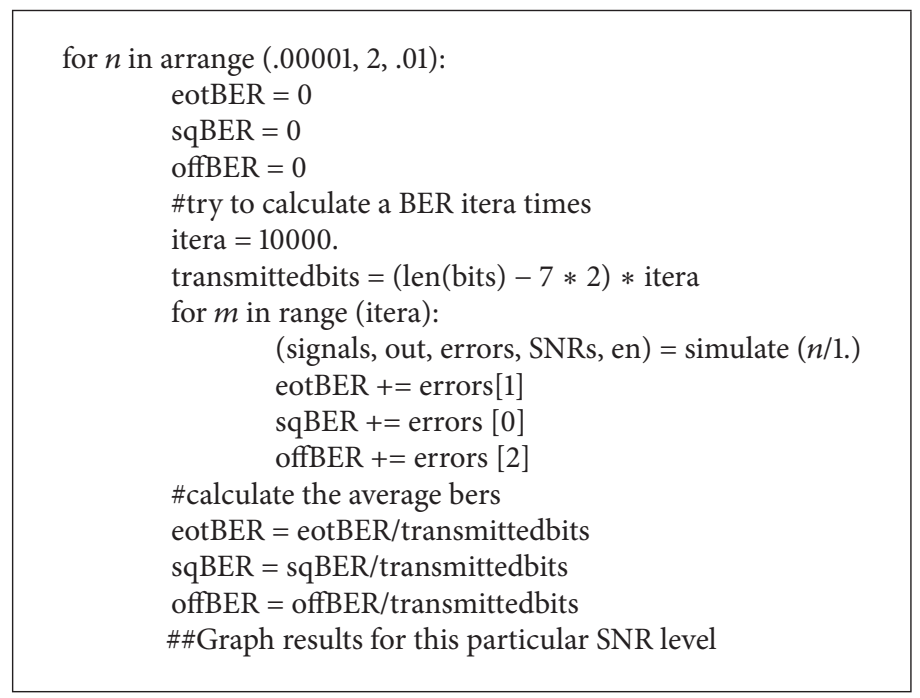

Pseudocode 1: Pseudocode illustrating BER versus SNR calculation.

the number of errors, resultant bit patterns, and energy, similar to the first simulate function. We again will perform $10^{4}$ iterations with random bit sequences for each iteration and a 128 bit vector length. For each value of the RC constant between 0 and 1 (step size of .01) we will plot RC versus BER. Python pseudocode example in Pseudocode 2 demonstrates this setup.

\section{Edge-Only Transmission}

3.1. Method Overview. In this paper, we present a novel signaling method intended to perform well in the presence of ISI at data rates approaching $20 \mathrm{~Gb} / \mathrm{s}$. We propose a modulation method called edge-only transmission (EOT) that transmits a relatively narrow-band pulse when a rising or 


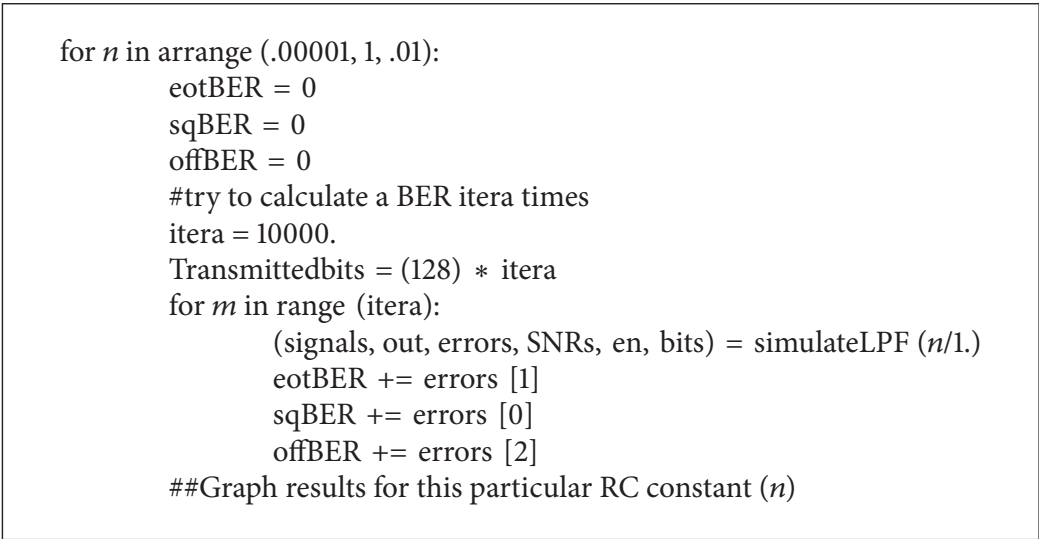

Pseudocode 2: Pseudocode illustrating BER versus ISI calculation.

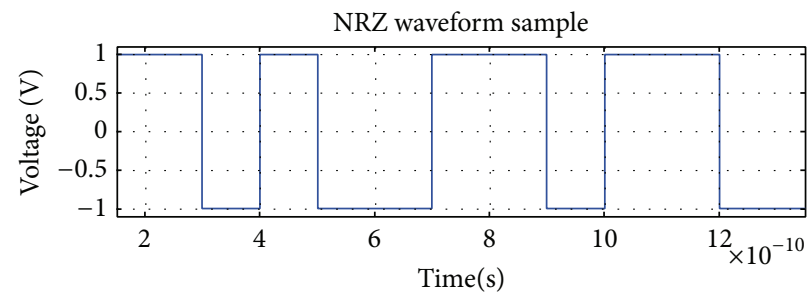

(a)

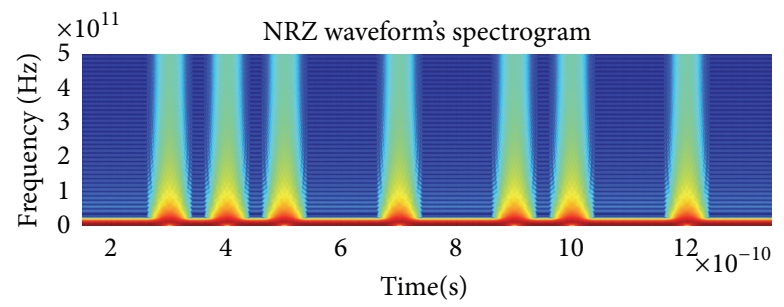

(b)

FIGURE 3: A square pulse signal (a) and its associated spectrogram (b).

falling edge occurs in a bit pattern. The EOT transmitter is a transformation of a square wave into a pulse edge-only signal. The resultant waveform will only transmit energy when an edge is detected in the data being sent. These signals will be transmitting pure edge information, similar to, for example, Manchester code [10] implementation.

A band-pass filter can be utilized to transmit the edge information and drop the nonedge values of the incoming NRZ signal as desired. To illustrate the technique we generated a spectrogram using the short-time Fourier transform method, which breaks a time-domain signal up into bins and performs an FFT on each. The frequency-normalized spectrogram was generated with the Matplotlib [11] spectrogram function. The input square wave is shown in Figure 3 along with its time-varying spectrum.

It is clear that the bulk of frequency components are concentrated around a bit transition, which can only occur at an edge. We can surmise then that the "true" information in a square-pulse signal is, indeed, contained in the edges only. If our filter is designed to drop the low-frequency components,

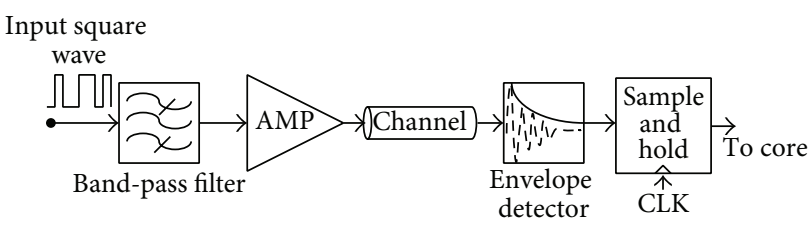

FIGURE 4: Block diagram of EOT transceiver implementation.

then the resulting signal will only have energy at times when an edge occurred. Thus, the presence or absence of a pulse of energy in the transmitted signal can communicate whether or not an edge was present in the original input; during signal recovery, a detected pulse is indicative of an edge. If the first bit state is known, a stateful receiver can keep track of when a bit flip occurs and flip the stored bit.

The block diagram of a transceiver assembly for EOT is shown in Figure 4. 


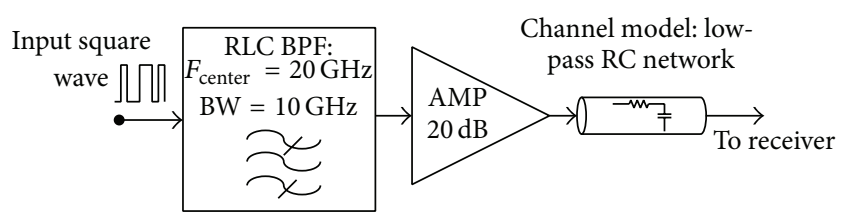

FIGURE 5: Block diagram of EOT transmission scheme model.

The only active component required in this modulation scheme is a relatively narrow-band amplifier, for example, described in [12], to preamplify the signal before transmission. The amplification is necessary since the band-pass filter is passive and thus dissipates energy. The amplification stage to the previous signal strength will also amplify any incident noise and makes apparent the loss of SNR. However, such an amplifier can be relatively easily designed at low cost and as a result the scheme is still cost effective.

3.2. Transmitter Implementation. For the transmitter we must choose a filter bandwidth and a center frequency to pass the signal through. For simulation, a second-order RLC circuit was used, with center frequency dependent on locality of ideal channel resonance. The result is then amplified. As the bandwidth is inversely proportional to the trail-off time of the generated pulse incident to the edge, we immediately see how the filter/amplifier combined bandwidth will determine how closely the symbols can be packed, as the ISI depends on the trail-off time as well. The setup shown in Figure 5 was used in simulation.

The signal was simulated to pass through a modeled channel with a transfer characteristic defined by $1 /(1+j \omega \mathrm{RC})$. The bandwidth and center frequency of EOT pulses were chosen arbitrarily to be $5 \mathrm{GHz}$ and $25 \mathrm{GHz}$, respectively. In initial simulation with random bits being transmitted at $2 \mathrm{~Gb} / \mathrm{s}$, Figure 6 shows the transmitted signal inserted into the channel, with center frequency $f_{0}=25 \mathrm{GHz}$ and bandwidths of $5 \mathrm{GHz}$ and $15 \mathrm{GHz}$.

This transmitter concept can be used in square-pulse legacy systems as an add-on. No additional logic is necessary for its implementation as it uses incoming square pulses as input. In low-loss environments, the BPF and amplifier can be easily bypassed, thus still maintaining good compatibility with legacy platforms. Finally, although not explicitly analyzed in this paper, it may be inferred that since BPF's center frequency is adjustable, the designer may deliberately choose different frequencies for different interfaces, thereby significantly reducing the crosstalk between them.

3.3. Receiver Implementation. To recover the transmitted symbols, we note that the energy received will be concentrated around the ideal edge durations. Thus we can perform energy detection by separating the signal into bins with time durations equal bit width and detecting a 1 if and only if a particular bin gathered enough energy to be above a detection threshold. The simulated receiver apparatus is shown in the block diagram in Figure 7.
The energy detector will have an implementation-specific threshold for detection which depends on the amount of energy expected to be transmitted and the available hardware. This receiver concept can be used in legacy systems intended for square-pulse signaling, as its output is a conventional square-pulse waveform.

3.4. ISI Effects on Signaling for EOT. EOT transmits the output of a band-pass filter (BPF). Let the bandwidth of the BPF be $B$ with center frequency $f_{0}$. Then the resulting transmitted signal will only be affected by channel loss in the frequency range $f_{0} \pm B$. For sufficiently small $B$, we can use a linear approximation of the RC-network's transfer function to show that the amplitudes of the spectra are scaled and not changed with respect to one another. Figure 8(a) shows the spectrum of an EOT signal with carrier frequency of $25 \mathrm{GHz}$ and an RLC transfer function overlaid. Figure 8(b) displays a close-up of the boxed part of the spectrum shown in Figure 8(a). We can see that within this area, the transfer function is approximately linear. As the bandwidth $B$ decreases this approximation becomes more accurate and thus the result of low-pass filtering the transmitted EOT waveform for $B \ll$ the RC circuit's bandwidth is equivalent to a scaling of the original signal. These results intuitively suggest that the signal is resilient to the effects of ISI, as an amplification stage at the receiver would be able to reverse the effects of the linear scaling done by the channel. The required amplification stage would however amplify any additive channel noise; therefore the process of linear approximation and receiver amplification will decrease the SNR. These results are discussed in Section 4.

\section{System Performance Modeling and Comparison}

In this section we will implement and compare EOT, OOK, and normal square wave modulation with eye-diagram and matched filter receivers using the benchmark methods outlined in Section 2. Two simulate( $n$ ) methods are used, one for BER versus ISI and the other for BER versus SNR.

The first benchmark to be performed is BER versus ISI. The cause for ISI degradation is assumed to be RCnetwork modeling low-pass characteristics of a typical wireline interconnect. The data rate is set to $10 \mathrm{~Gb} / \mathrm{s}$ and the EOT resonance frequency and bandwidth are $20 \mathrm{GHz}$ and $10 \mathrm{GHz}$, respectively. The OOK transmission used a carrier frequency equal to the EOT resonance frequency. The RC factor was evaluated over a range of 0 to 1 with a step size of .01 and $10^{4}$ iterations were run for each $\mathrm{RC}$ value with a different random bit sequence used each time. The total BER was calculated and Figure 9 displays the result. The EOT performs better than matched filter (MF) receiver and better than eye-based receiver, both with NRZ pulses. The integrated OOK receiver outperforms all signaling/receiver types by an even wider margin. Square wave signaling with a matched filter (MF) performs better at extreme values of the RC constant as a single missed bit using EOT results in the rest of that bit sequence being read incorrectly. However, EOT still performs 

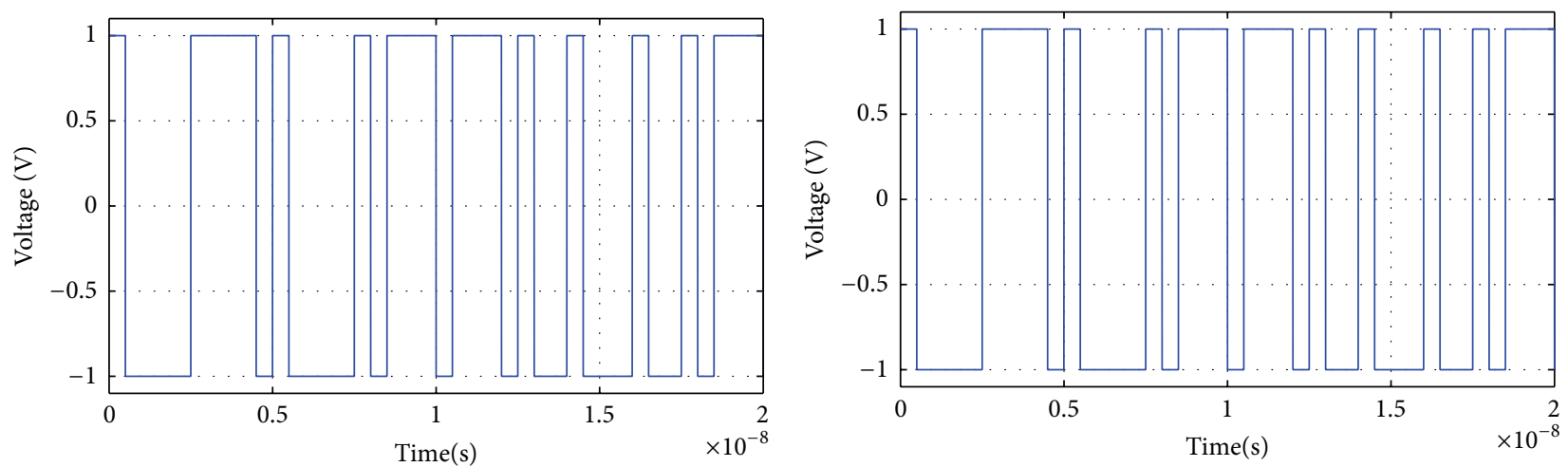

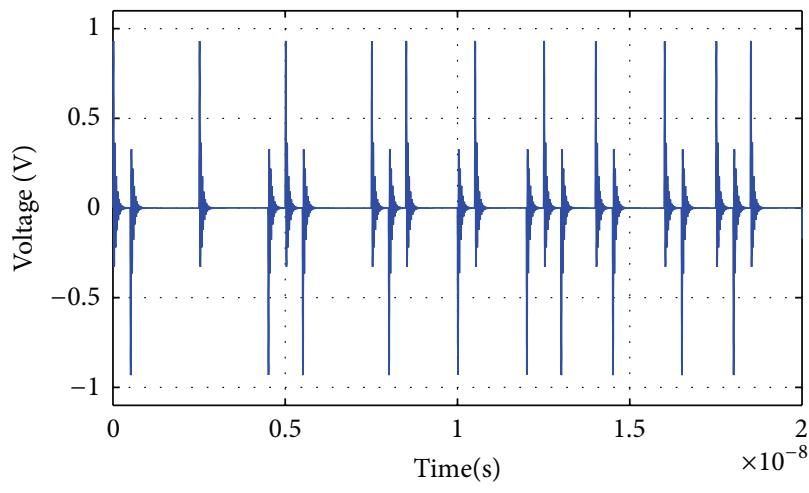

(a)

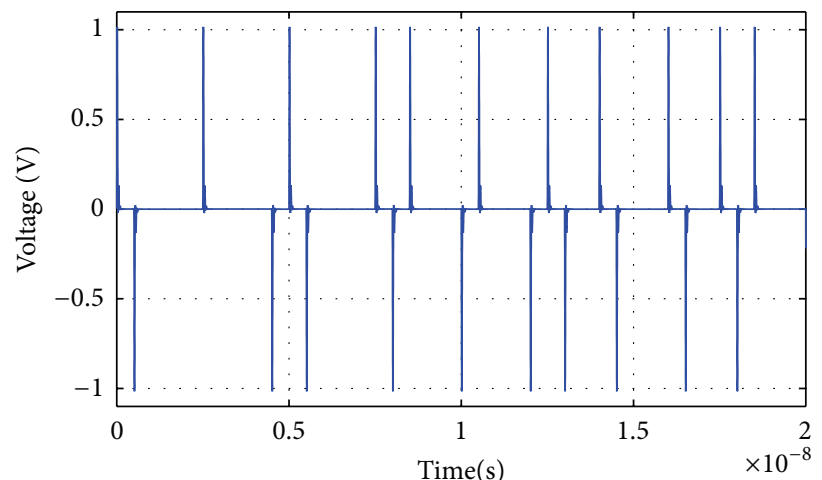

(b)

Figure 6: Input signals and (a) $5 \mathrm{GHz}$ bandwidth EOT and (b) $15 \mathrm{GHz}$ bandwidth EOT.

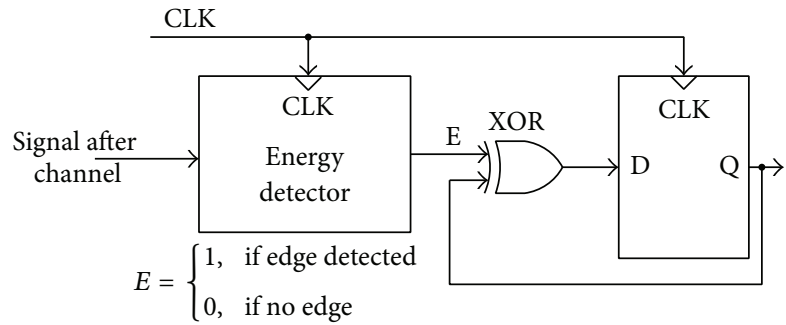

Figure 7: Block diagram of EOT receiver implementation.

better in the range from $\mathrm{RC}=0.1$ to $\mathrm{RC}=0.13$ for a fixed bandwidth. No error correction or detection methods were used in conjunction with EOT; however, it is evident that using these methods will improve EOT's performance even more.

The same setup was again used to generate a benchmark in the presence of noise, by comparing the BER of the signal at a given SNR. This time the simulate $(n)$ method was used with $n$ as the additive noise constant $C$, and the resulting BER was plotted against the SNR. The results are shown in Figure 10. The matched filter (MF) receiver with the square wave input exhibits the best performance, as expected, followed by OOK and EOT, with eye-based receivers performing the worst. In the presence of noise EOT performs not as well as the other alternative methods because any bit error caused by a random noise spike will cause the rest of the sequence to be read incorrectly. However, at SNR levels above $4 \mathrm{~dB}$ the scheme still performs well and outperforms eye-based signaling, and thus it is still well suited for operation in environments with SNR greater than around $3 \mathrm{~dB}$ at $10 \mathrm{~Gb} / \mathrm{s}$. Again, any error correction/control methods used in conjunction with EOT are expected to greatly improve the method's performance, which is the topic of future study.

While our work focuses on top-level system performance characteristics, such as ISI and BER effects, we can also make preliminary inferences regarding implementation of the proposed scheme in actual integrated circuits. One such parameter of interest is power consumption. It is recognized that the EOT method requires a low noise amplifier (LNA) operating in the $\mathrm{GHz}$ range; there is a wide body of work dedicated to the development of such circuits, and we cited only one example [12] which demonstrates availability of low-power LNA technology suitable for implementation. Obviously, power consumption is a function of a particular scenario in which a circuit is to be used; however, we can still make a qualitative comparison between EOT power requirements and that of other considered methods, based on the LNA in [12]; the reported power consumption at supply voltage of $1.2 \mathrm{~V}$ and $130-\mathrm{nm}$ process was $24 \mathrm{~mW}$ per differential interface (or $12 \mathrm{~mW}$ per leg). This would correspond to a required current of $10 \mathrm{~mA}$ in a typical differential driver (without a high-frequency amplifier) intended for high-speed 


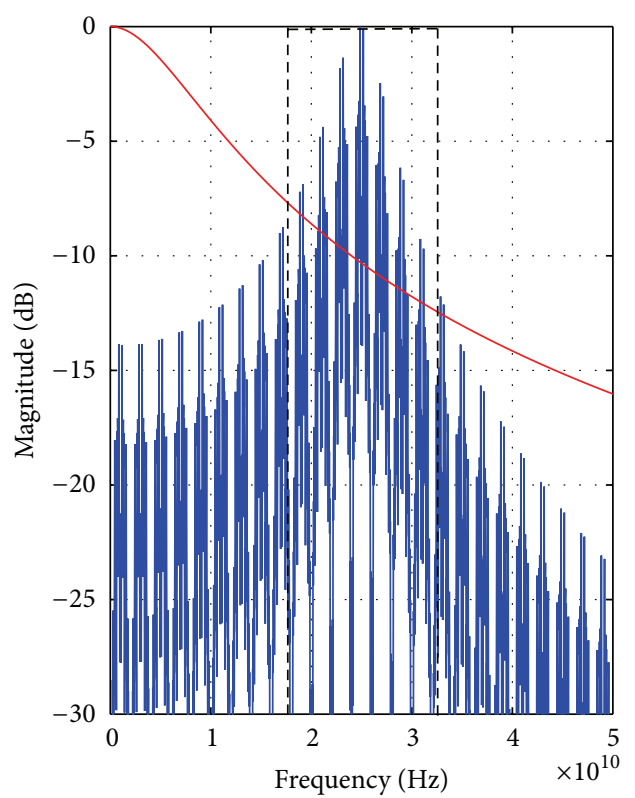

(a)

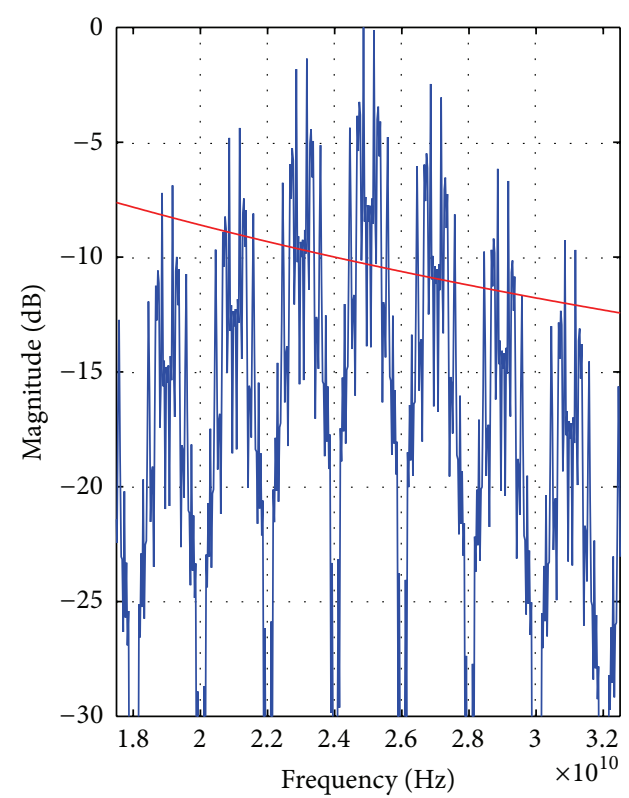

(b)

FIgURE 8: Spectrum of an EOT signal and a low-pass transfer function. The boxed area in (a) indicates the part of the EOT spectrum which contains most of its energy and (b) shows the close-up of this area.

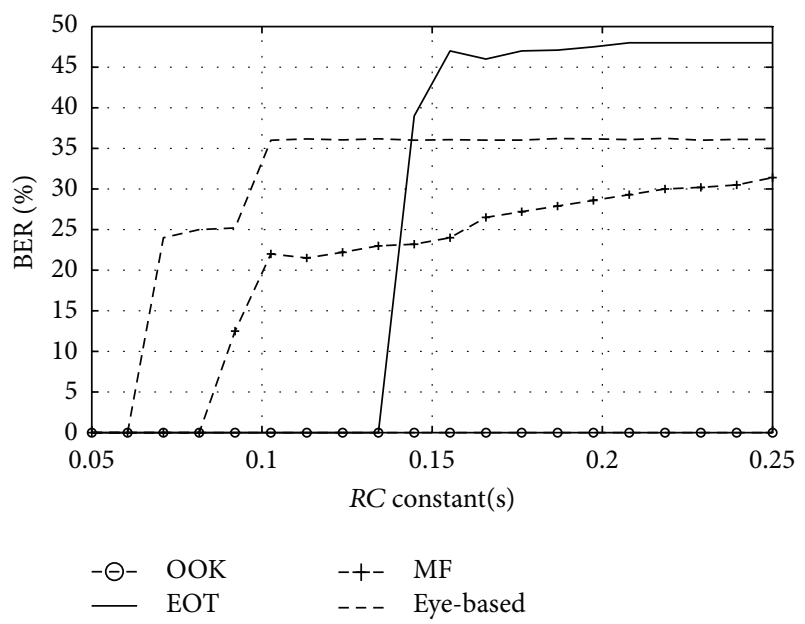

FIGURE 9: BER as a function of RC-induced ISI.

serial data transmission. In our experience, this current value is at a lower end of requirements for general implementations intended for driving copper backplane traces 5-20 inches in length. Thus, without performing a detailed analysis, we can still surmise that power consumption of the proposed scheme that lacks a relatively high-power driver but contains a bandpass amplifier appears to be roughly on a par with traditional implementations of square-pulse signaling methods based upon differential drivers. Finally, although not explicitly analyzed in this paper, it may be inferred that since BPF's center frequency is adjustable, the designer may deliberately choose different frequencies for different interfaces, thereby significantly reducing the crosstalk between them; also,

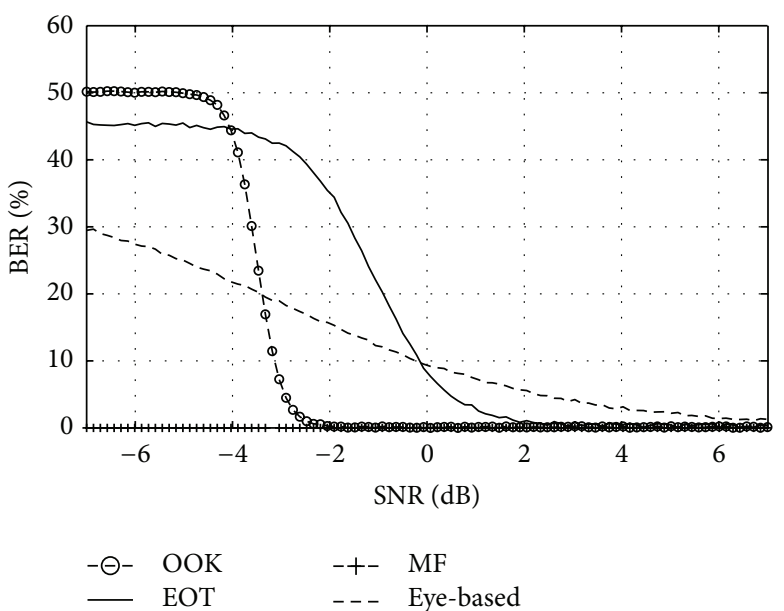

FIGURE 10: BER as a function of SNR.

higher center frequencies may be utilized to excite higher modes of propagation, which may reduce the amount of loss encountered, for example, in strip-line structures [13].

\section{Conclusion}

Square pulses are very wideband, making them ineffective in the environments with high levels of ISI. Although a matched filter will detect energy leaked from previous symbols, it will do better detecting transmitted bits which only have errors in a limited area or for a limited duration. EOT removes channel-dependent ISI almost entirely if a transmitter amplifier with sufficient bandwidth is used, and it 
is clearly more optimal in high-speed configurations where ISI is prevalent. Sophisticated methods of equalizing lossy channels are reported in the literature as being able to extend the data transfer speeds to $10-20 \mathrm{~Gb} / \mathrm{s}$, while requiring tapped delay lines and FIR filter implementations. EOT, on the other hand, does not require delay lines and can be implemented with a single RCL BPF, an amplifier and a relatively simple and area-efficient logic in the receiver. Initial power analysis shows that the trade-off of a high-power driver (needed for typical square-pulse transmission) and a lownoise amplifier (needed for EOT) is not likely to dramatically increase the power consumption of the proposed scheme and is more likely to result in about equal power values. EOT performs well in the presence of ISI due to its relatively narrow bandwidth, and by setting a threshold value and amplification level for a particular setup the BER can be met without complicated channel equalization necessary for line-encoded square waves. OOK performs the best, utilizing an integrator and not relying on energy near DC as square wave signaling does. Like EOT, it can also have a variable threshold value to help increase the BER. However, in contrast to OOK, EOT requires only minimal modification to existing wireline transmission topologies and can be easily used in legacy platforms. The OOK transmission requires a high-speed oscillator and a synchronized clock, which may be difficult to implement and have little to no availability present at comparable cost. EOT is able to function well in environments with a $3 \mathrm{~dB}$ or higher SNR level and is cost effective. This combined with the similarities between conventional methods makes it an effective choice for nextgeneration hardware at higher signaling rates. An intriguing possibility afforded by usage of a LNA/BPF combination in EOT can be in choosing the center frequency in such a way as to excite higher propagation modes, thus dramatically reducing propagation loss, which, in our opinion, warrants future work as well.

\section{Conflict of Interests}

The authors declare that there is no conflict of interests regarding the publication of this paper.

\section{References}

[1] J. Liu and X. F. Lin, "Equalization in high-speed communication systems," IEEE Circuits and Systems Magazine, vol. 4, no. 2, pp. 4-17, 2004.

[2] R. Payne, P. Landman, B. Bhakta et al., "A 6.25-Gb/s binary transceiver in $0.13-\mu \mathrm{m}$ CMOS for serial data transmission across high loss legacy backplane channels," IEEE Journal of Solid-State Circuits, vol. 40, no. 12, pp. 2646-2657, 2005.

[3] T. M. Hollis, D. J. Comer, and D. T. Comer, "Mitigating ISI through self-calibrating continuous-time equalization," IEEE Transactions on Circuits and Systems I: Regular Papers, vol. 53, no. 10, pp. 2234-2245, 2006.

[4] H. Kim, F. Bien, Y. Hur et al., "A 0.25-um BiCMOS feed foward equalizer using active delay line for backplane communication," in Proceeding of the IEEE International Symposium on Circuits and Systems (ISCAS '07), pp. 193-196, New Orleans, La, USA, May 2007.

[5] S. Pavan and R. Tiruvuru, "Analysis and design of singly terminated transmission-line FIR adaptive equalizers," IEEE Transactions on Circuits and Systems I: Regular Papers, vol. 54, no. 2, pp. 401-410, 2007.

[6] C. Pelard, E. Gebara, A. J. Kim et al., "Realization of multigigabit channel equalization and crosstalk cancellation integrated circuits," IEEE Journal of Solid-State Circuits, vol. 39, no. 10, pp. 1659-1670, 2004.

[7] Equalizing Gigabit-per-Second Signals on Copper Media, Application note HFAN-06.0.1, Rev. 2, Maxim Integrated Products, Sunnyvale, Calif, USA, 2008.

[8] Understanding and Characterizing Timing Jitter Primer, Tektronix, 2012, http://www.tek.com/application/jitter-measurement-and-timing-analysis.

[9] N. Kim, M. Sung, H. Kim et al., "Reduction of crosstalk noise in modulator Jack for high-speed differential signal interconnection," IEEE Transactions on Advanced Packaging, vol. 24, no. 3, pp. 260-267, 2001.

[10] G. Fairhurst, “Manchester Encoding, Tutorial," University of Aberdeen, Aberdeen, UK, http://www.erg.abdn.ac.uk/ gorry/ course/phy-pages/man.html.

[11] Spectrogram (Signal Processing Toolbox), MATLAB Help files, The MathWorks, Natick, Mass, USA, 2006.

[12] G. Xiaoling and K. O. Kenneth, "A power efficient differential 20-GHz low noise amplifier with 5.3-GHz 3-dB bandwidth," IEEE Microwave and Wireless Components Letters, vol. 15, no. 9, pp. 603-605, 2005.

[13] F. Kuroki, K. Miyamoto, and S. Nishida, "Transmission characteristics of the first higher mode in a strip line at millimeterwavelength," in Proceedings of the IEEE MTT-S International Microwave Symposium, pp. 1901-1904, Long Beach, Calif, USA, June 2005. 

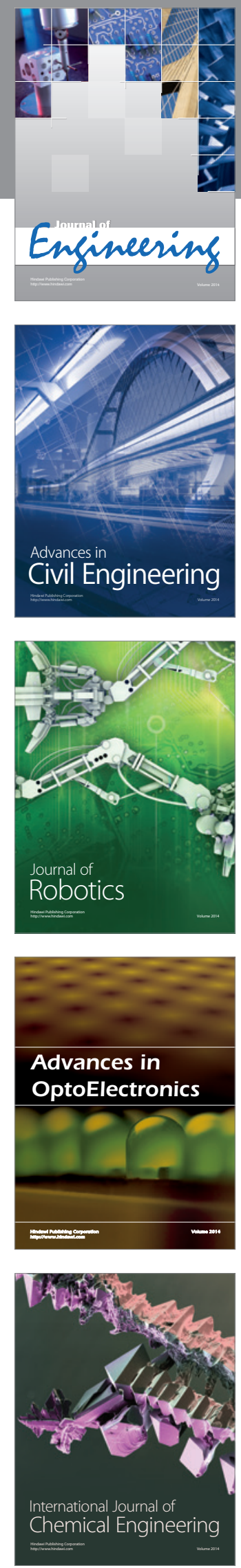

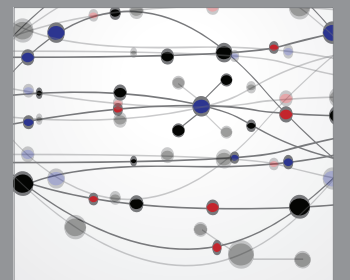

The Scientific World Journal
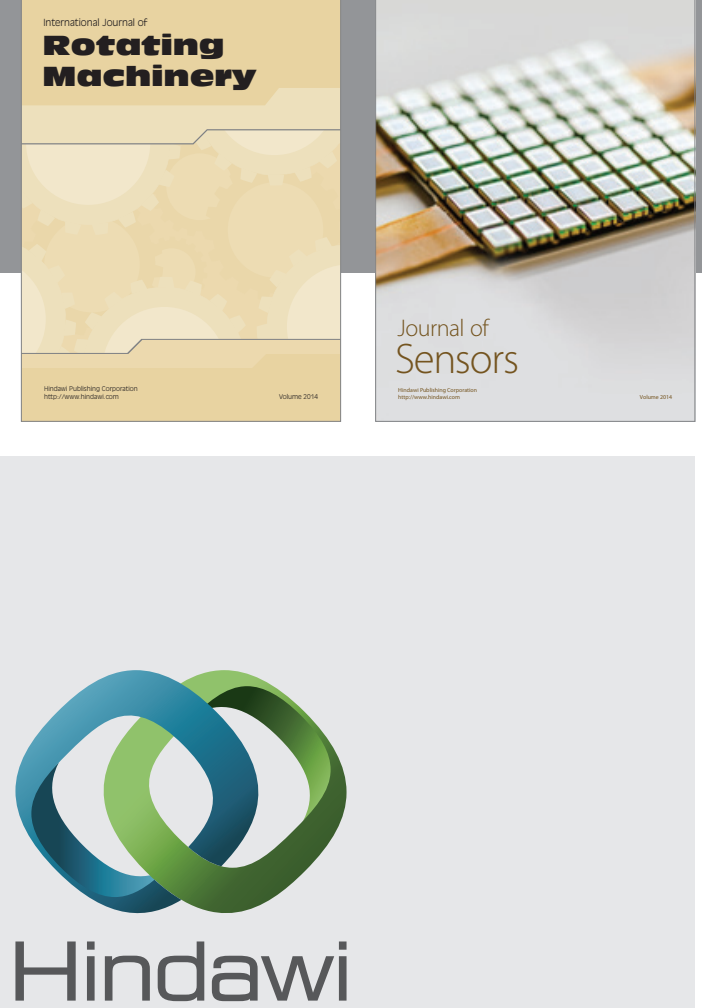

Submit your manuscripts at http://www.hindawi.com
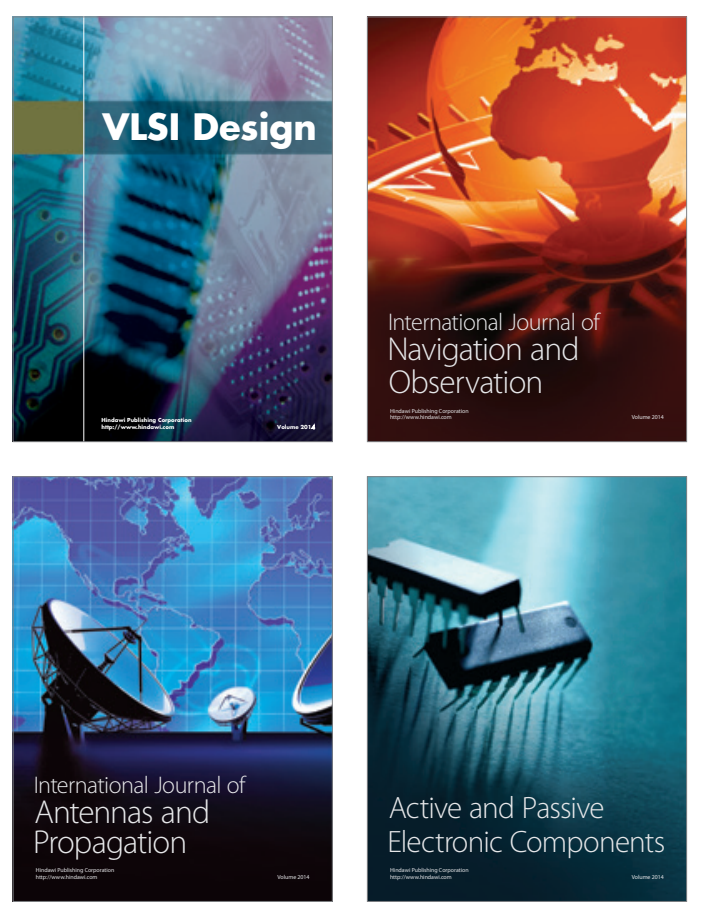
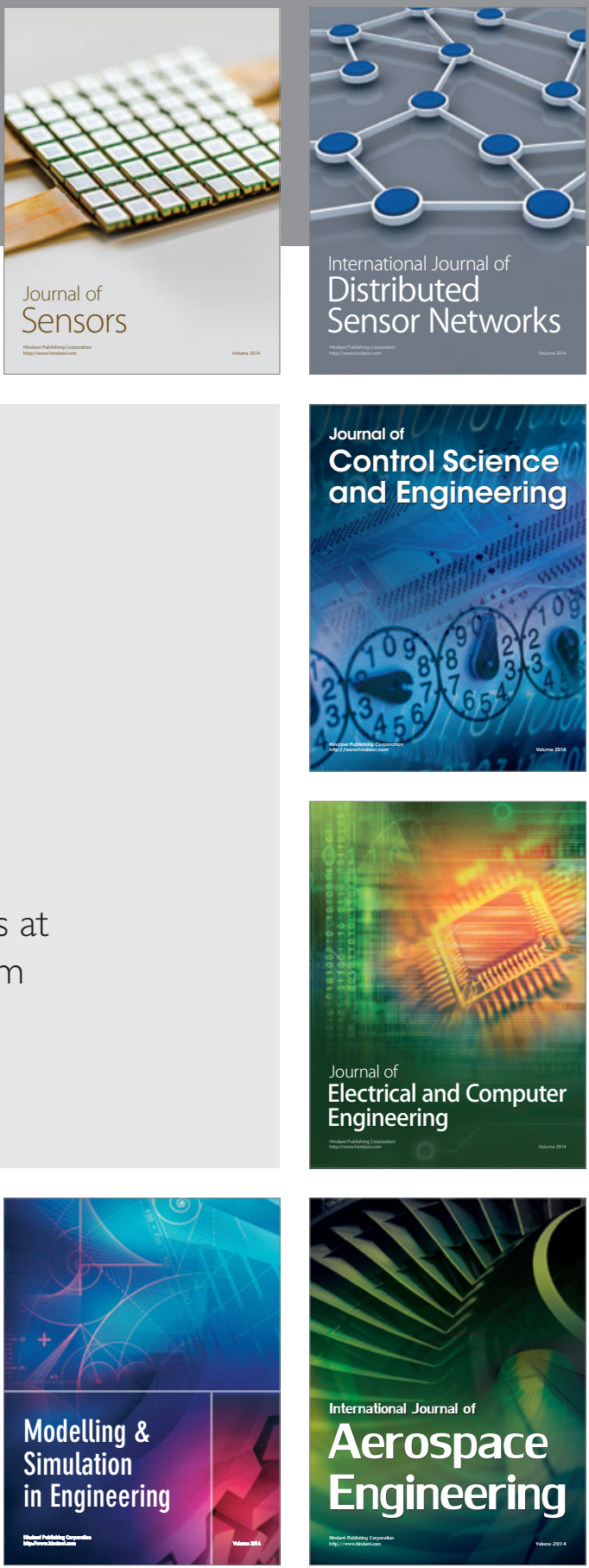

Journal of

Control Science

and Engineering
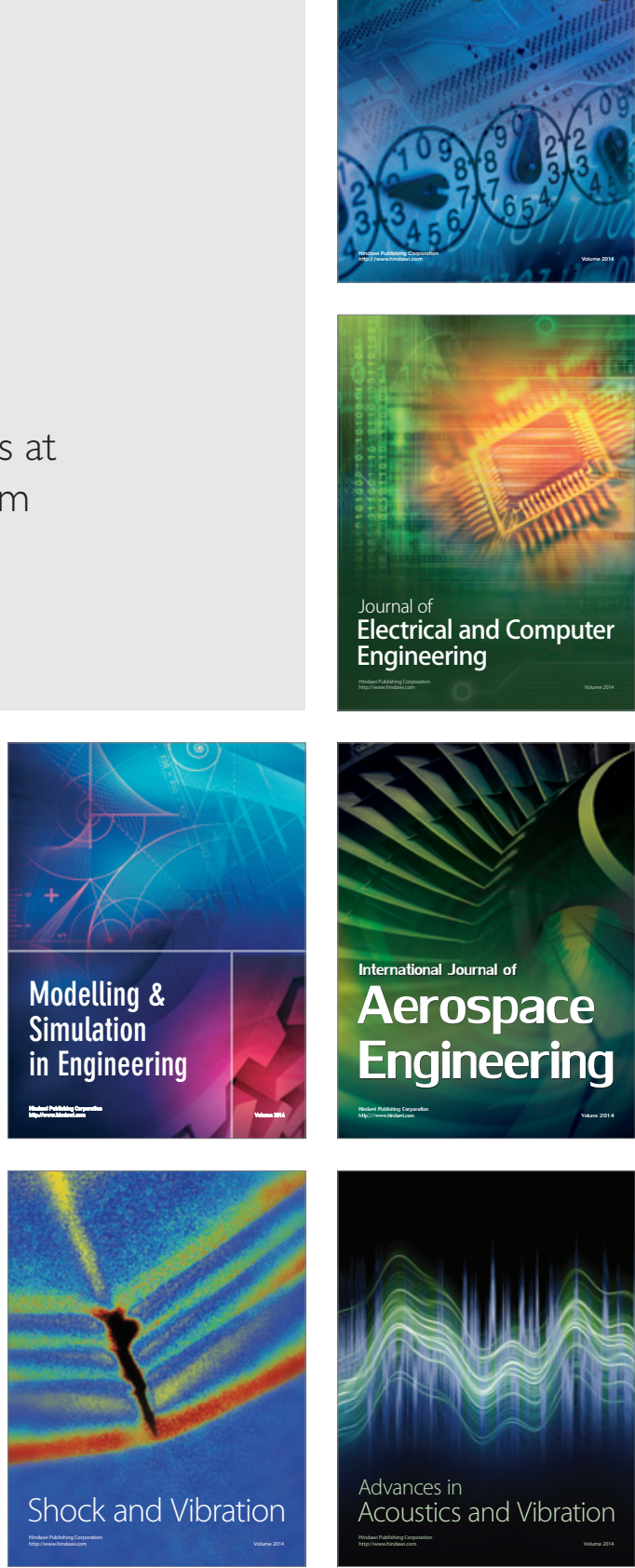